\title{
Elliptical micropillar cavity design for highly efficient polarized emission of single photons
}

Gür, Uur Meriç; Mattes, Michael; Arslanagi, Samel; Gregersen, Niels

Published in:

Applied Physics Letters

Link to article, DOI:

$10.1063 / 5.0041565$

Publication date:

2021

Document Version

Peer reviewed version

Link back to DTU Orbit

Citation (APA):

Gür, U. M., Mattes, M., Arslanagi, S., \& Gregersen, N. (2021). Elliptical micropillar cavity design for highly efficient polarized emission of single photons. Applied Physics Letters, 118(6), [061101].

https://doi.org/10.1063/5.0041565

\section{General rights}

Copyright and moral rights for the publications made accessible in the public portal are retained by the authors and/or other copyright owners and it is a condition of accessing publications that users recognise and abide by the legal requirements associated with these rights.

- Users may download and print one copy of any publication from the public portal for the purpose of private study or research.

- You may not further distribute the material or use it for any profit-making activity or commercial gain

- You may freely distribute the URL identifying the publication in the public portal

If you believe that this document breaches copyright please contact us providing details, and we will remove access to the work immediately and investigate your claim. 


\title{
Elliptical micropillar cavity design for highly efficient polarized emission of single photons
}

\author{
Uğur Meriç Gür, ${ }^{1}$ Michael Mattes, ${ }^{1}$ Samel Arslanagić, ${ }^{1}$ and Niels Gregersen ${ }^{2}$ a) \\ ${ }^{1)}$ Department of Electrical Engineering, Technical University of Denmark, Ørsteds Plads, Building 348, 2800 Kongens Lyngby, \\ Denmark \\ ${ }^{2)}$ Department of Photonics Engineering, Technical University of Denmark, Ørsteds Plads, Building 343, 2800 Kongens Lyngby, \\ Denmark
}

(Dated: 10 February 2021)

We present a numerical investigation of the performance of the micropillar cavity single-photon source featuring an elliptical cross-section. While ellipticity serves to establish polarization control of the emitted photons, it introduces a trade-off due to reduced coupling to a Gaussian profile in the far field as well as the need for significantly increased pumping power when a resonant excitation pumping scheme is implemented. Starting from a circular micropillar, we identify optimum geometrical parameters leading to a maximum polarized coupling efficiency of 0.90 to a Gaussian profile in the far field combined with a modest relative increase in pump power of $16 \mathrm{~dB}$.

Within optical quantum information processing, quantum bits are encoded on single photons, and thus a central building block of associated systems is the single-photon source ${ }^{1}$ (SPS). Its figures of merit ${ }^{2}$ are the efficiency $\varepsilon$ defined as the number of photons detected by the collection optics per trigger, and the indistinguishability $\eta$ of the emitted photons, as well as their purity $g^{(2)}(\tau=0)$. The success probability $P$ of a multi-photon interference experiment with $N$ photons scales as $P=(\varepsilon \eta)^{N}$, and increasing $\varepsilon \eta$ is thus vital in the realization of scalable quantum information technologies. Whereas the spontaneous emission down-conversion ${ }^{3}$ process has been a major workhorse within quantum optics for the past three decades, its probabilistic nature limits the efficiency to around $\sim 0.01$. For this reason, an alternative strategy for deterministic single-photon emission based on two-level systems ${ }^{4}$ has been pursued, and within the last decade the semiconductor quantum $\operatorname{dot}^{5}$ (QD) embedded in a host material has emerged as a dominating platform for efficient generation of single indistinguishable photons.

For a QD in a bulk material, the large index contrast at the semiconductor-air interface ${ }^{6}$ leads to a low collection efficiency, and it is necessary to structure the photonic environment to direct the emitted photons towards the collection optics. ${ }^{1}$ The relatively large penetration of light into metals at optical frequencies and the associated loss have motivated the development of two classes of all-dielectric SPS designs; narrow-band designs such as the micro-cavity pillar, ${ }^{7-18}$ where the light emission is controlled using cavity quantum electrodynamics effects, and broad-band designs including the photonic nanowire, ${ }^{19,20}$ the photonic crystal waveguide ${ }^{21}$ and the microlens ${ }^{22}$, where the emission is controlled using a combination of dielectric screening, slow-light and classical beam shaping effects.

For sources featuring vertical emission of light, the micropillar cavity depicted in Fig. 1 consisting of a $\lambda$ (wavelength) cavity sandwiched between two distributed Bragg reflectors (DBRs) is presently the champion SPS design: Using the resonant excitation ${ }^{23}$ pumping scheme, indistinguish-

\footnotetext{
${ }^{a)}$ Author to whom correspondence should be addressed: ngre@ fotonik.dtu.dk
}

able photon emission with $\eta \sim 0.99^{7,8}$ combined with efficiency up to $\varepsilon \sim 0.33^{7}$ has been demonstrated for rotationally symmetric micropillars. While resonant excitation avoids the time-jitter ${ }^{24}$ detrimental to the indistinguishability of nonresonant excitation schemes, it introduces the necessity to filter out the pump laser light typically using a cross-polarization setup. This passive polarization filtering, which also serves to project the light into a single polarization as required by most applications, limits the maximum achievable efficiency to $50 \%$ for the rotationally symmetric micropillar ${ }^{9}$. Alternative pumping techniques avoiding this barrier include twocolor resonant excitation, ${ }^{10}$ which however puts a limit on the cavity Q factor, and phonon-assisted excitation, where a lower indistinguishability of $\eta \sim 0.91$ was recently demonstrated. ${ }^{11}$

One strategy for overcoming the $50 \%$ barrier of the resonant excitation scheme is to lift the spectral degeneracy of

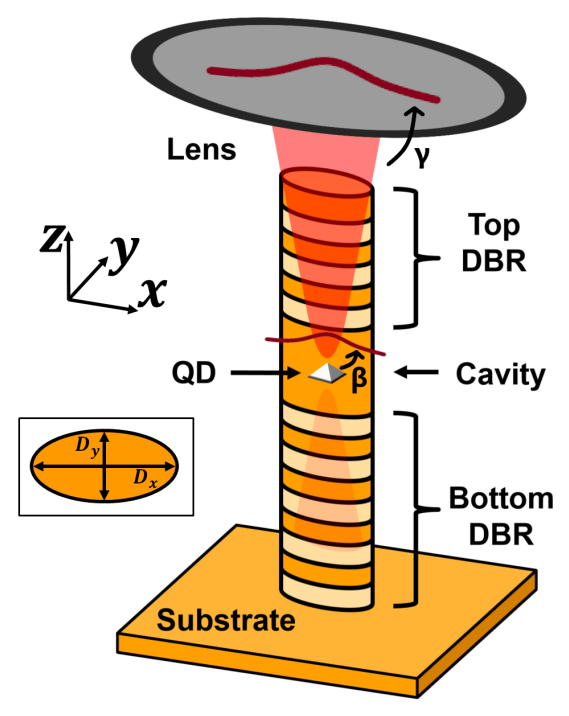

FIG. 1. The elliptical micropillar SPS, where the QD is placed in the center of a $\lambda$ cavity surrounded by distributed Bragg reflectors (DBRs) with $N_{\text {top }}\left(N_{\text {bottom }}\right)$ layer pairs in the top (bottom) mirror. The inset shows the cross-section featuring a major $\left(D_{x}\right)$ and the minor $\left(D_{y}\right)$ axis. 
the two orthogonally polarized $\mathrm{X}$ and $\mathrm{Y}$ cavity modes using an elliptical cross-section ${ }^{12-16}$. Here, the QD is spectrally aligned with e.g. the co-polarized $\mathrm{X}$ mode, and since the cross-polarized $\mathrm{Y}$ mode is spectrally detuned, photons will be preferentially emitted into the co-polarized $\mathrm{X}$ mode. This scheme was recently exploited to demonstrate ${ }^{12}$ an efficiency $\varepsilon$ of 0.6 combined with $\eta \sim 0.97$, thus overcoming the $50 \%$ barrier of the cross-polarization scheme.

The increasing ellipticity leads to a reduction in the coupling to a Gaussian profile in the far field and also an increase in the required pump power, as the QD is pumped using the cross-polarized misaligned Y mode. However, the corresponding limits to the achievable polarization control, and in turn the total efficiency, as function of ellipticity, spectral shift and cavity Q factor have not yet been explored. A recent work presents the numerical optimization ${ }^{17}$ of the circular micropillar cavity in the presence of phonon-induced decoherence ${ }^{24}$ resulting from the interaction with lattice vibrations of the solid-state environment, and here an optimum performance of 0.95 unpolarized efficiency combined with 0.997 indistinguishability was demonstrated. We select the optimum circular geometry and then introduce a gradual ellipticity preserving the indistinguishability. We subsequently perform a numerical optimization of the achievable efficiency from a micropillar cavity featuring an elliptical cross-section demonstrating enhanced performance characteristics.

We consider the micropillar cavity structure shown in Fig. 1. Following Ref. 17, the vertical cavity is formed by two asymmetrical distributed Bragg reflectors made from alternating layers of $\mathrm{GaAs}$ and $\mathrm{Al}_{0.85} \mathrm{Ga}_{0.15}$ As with $N_{\text {top }}\left(N_{\text {bottom }}\right)$ periodic layer pairs in the top (bottom) mirror. The refractive indices are 3.5015 and 2.9982 for $\mathrm{GaAs}$ and $\mathrm{Al}_{0.85} \mathrm{Ga}_{0.15} \mathrm{As}$ layers ${ }^{25}$, respectively, and the free-space design wavelength is $\lambda_{0}=895 \mathrm{~nm}$. The condition $N_{\text {bottom }}>N_{\text {top }}$ should always be met to avoid leakage of the electromagnetic field into the substrate. The thicknesses of the DBR layers are chosen such that reflected waves interfere constructively, meaning that each layer has a thickness of $\lambda_{0} /\left(4 n_{\text {eff }}\right)$, where $n_{\text {eff }}$ is the effective refractive index of the fundamental mode in the corresponding layer. The one wavelength-thick cavity $\left(\lambda_{0} / n_{\text {eff }}\right)$ leads to an anti-node at the QD position in the center of the cavity. While the mirror structures lead to optical confinement in the vertical direction, the lateral confinement is ensured via total internal reflection at the semiconductor-air interface. Finally, we consider a collection lens with a numerical aperture of 0.82 placed in the far field.

The nanophotonic environment is analyzed with the open geometry modal method introduced in Refs. 26-28, for orthogonal curvilinear coordinate systems. Here, we divide the computational domain into uniform layers along the vertical propagation $z$ axis and expand the fields in forward and backward propagating eigenmodes as

$$
\begin{aligned}
\mathbf{E}\left(\mathbf{r}_{\perp}, z\right) & =\sum_{j} a_{j} \mathbf{e}_{j}\left(\mathbf{r}_{\perp}\right) e^{i \beta_{j} z}+\sum_{j} b_{j} \mathbf{e}_{j}\left(\mathbf{r}_{\perp}\right) e^{-i \beta_{j} z} \\
\mathbf{H}\left(\mathbf{r}_{\perp}, z\right) & =\sum_{j} a_{j} \mathbf{h}_{j}\left(\mathbf{r}_{\perp}\right) e^{i \beta_{j} z}+\sum_{j} b_{j} \mathbf{h}_{j}\left(\mathbf{r}_{\perp}\right) e^{-i \beta_{j} z}
\end{aligned}
$$

where $\mathbf{E}$ and $\mathbf{H}$ are the electric and the magnetic fields, re- spectively, in the transverse coordinates $\mathbf{r}_{\perp}, a_{j}$ and $b_{j}$ are the expansion coefficients for the $j$ th eigenmode, $\mathbf{e}_{j}$ and $\mathbf{h}_{j}$ are the eigenmodes, $\beta$ is the propagation constant, and $z$ is the longitudinal coordinate. The eigenmodes of interest and the associated propagation constants are determined by solving the Helmholtz's equation in the uniform layers. The spontaneous emission process of the QD is modeled ${ }^{29}$ using a classical point dipole, and the field expansion coefficients are determined by projecting ${ }^{17,30}$ the exciting current distribution (a point dipole with in-plane orientation) onto the eigenmodes. Subsequently, the reflection and transmission coefficients at the layer interfaces are calculated using S matrix theory ${ }^{30}$.

In the case of a charged exciton or a neutral exciton with a $45^{\circ}$ angle between the dipole and the $x$ and $y$ axes, the light emission is unpolarized ${ }^{9,31}$ and the $x / y$-polarized spontaneous emission rate is proportional ${ }^{29}$ to the total classical power $P_{\mathrm{T}}^{x / y}$, including the contribution to the radiation modes, emitted by an $x / y$-oriented classical dipole. Furthermore, since the emitter is placed at the (on-axis) center, an $x / y$-oriented dipole does not emit into the Y/X cavity mode. With these assumptions, the spontaneous emission $\beta^{\mathrm{X} / \mathrm{Y}}$ factor for the $\mathrm{X} / \mathrm{Y}$ cavity mode is given by

$$
\beta^{\mathrm{X} / \mathrm{Y}}=\frac{P_{\mathrm{cav}}^{\mathrm{X} / \mathrm{Y}}}{P_{\mathrm{T}}^{x}+P_{\mathrm{T}}^{y}}=\frac{F^{\mathrm{X} / \mathrm{Y}}}{P_{\mathrm{T}}^{x} / P_{\mathrm{bulk}}+P_{\mathrm{T}}^{y} / P_{\text {bulk }}},
$$

where $P_{\mathrm{cav}}^{\mathrm{X} / \mathrm{Y}}$ represents the power coupled into the fundamental cavity $\mathrm{X} / \mathrm{Y}$ mode from an $x / y$-oriented dipole. It is typically presented in terms of the Purcell factor ${ }^{32} F$ as ${ }^{29,33}$

$$
F^{\mathrm{X} / \mathrm{Y}}(\lambda)=\frac{P_{\text {cav }}^{\mathrm{X} / \mathrm{Y}}(\lambda)}{P_{\text {bulk }}(\lambda)},
$$

describing the wavelength-dependent power emission relative to the power $P_{\text {bulk }}(\lambda)$ emitted by the dipole into a bulk medium. While the maximum Purcell enhancement is obtained on-resonance, the Purcell factor for the off-resonant cross-polarized mode can also be defined through (4). The power $P_{\text {lens }}^{\mathrm{X} / \mathrm{Y}}$ collected by the lens in the far field is computed using a near-field to far-field transformation ${ }^{34}$ and an overlap with a Gaussian profile ${ }^{35,36}$ to model coupling to a singlemode fiber. Consequently, we define the transmission $\gamma^{\mathrm{X} / \mathrm{Y}}$ from the $\mathrm{X} / \mathrm{Y}$ cavity mode to the lens (cf. Fig. 1) as the ratio $\gamma^{\mathrm{X} / \mathrm{Y}}=P_{\text {lens }}^{\mathrm{X} / \mathrm{Y}} / P_{\mathrm{cav}}^{\mathrm{X} / \mathrm{Y}}$. The overall source efficiency $\varepsilon^{\mathrm{X} / \mathrm{Y}}$ is then obtained in a single-mode picture ${ }^{17}$ as the product of the spontaneous emission factor $\beta^{\mathrm{X} / \mathrm{Y}}$ and the collection efficiency $\gamma^{\mathrm{X} / \mathrm{Y}}$ such that $\varepsilon^{\mathrm{X} / \mathrm{Y}}=\beta^{\mathrm{X} / \mathrm{Y}} \gamma^{\mathrm{X} / \mathrm{Y}}$. Finally, we consider the quality factor $\mathrm{Q}$ for the $\mathrm{X}$ and $\mathrm{Y}$ cavity modes computed by constructing an eigenvalue problem using the cavity roundtrip matrix outlined in Ref. 37.

Our starting point is the optimized circular micropillar configuration presented in Ref. 17. The structure features a highreflectivity bottom mirror with $N_{\text {bottom }}=40$ DBR pairs and a top mirror with $N_{\text {top }}=21$ pairs with optimum $\varepsilon \eta$ performance obtained in the $D \in[2,4] \mu \mathrm{m}$ diameter range. The indistinguishability computed in Ref. 17 depends on the cavity $Q$ factor and the mode volume, and if these quantities are 


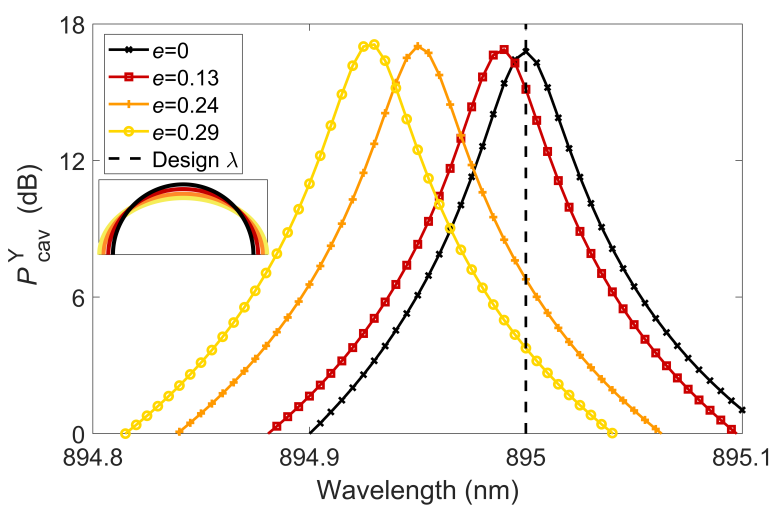

FIG. 2. The emission of the fundamental cavity cross-polarized $Y$ mode in the elliptical micropillar structure. Each curve represents the cross-polarization emission as a function of wavelength for different ellipticities $(e)$. The sum of the length of the major and the minor axis is constant, $D_{x}+D_{y}=6 \mu \mathrm{m}$. The black curve represents a circular micropillar, whereas the yellow represents the largest ellipticity. The half cross-sections are shown in the inset with matching colors. The thicknesses of the DBR layers are always chosen such that the copolarization X mode operates at the design wavelength of $895 \mathrm{~nm}$. (a)

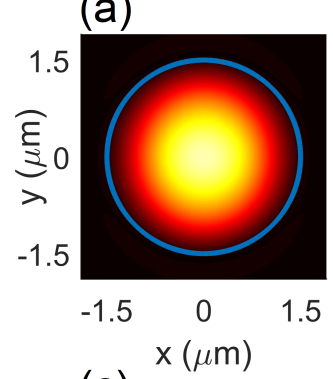

(c)

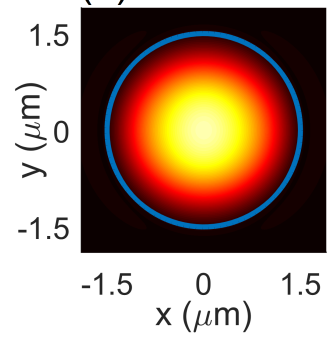

(b)

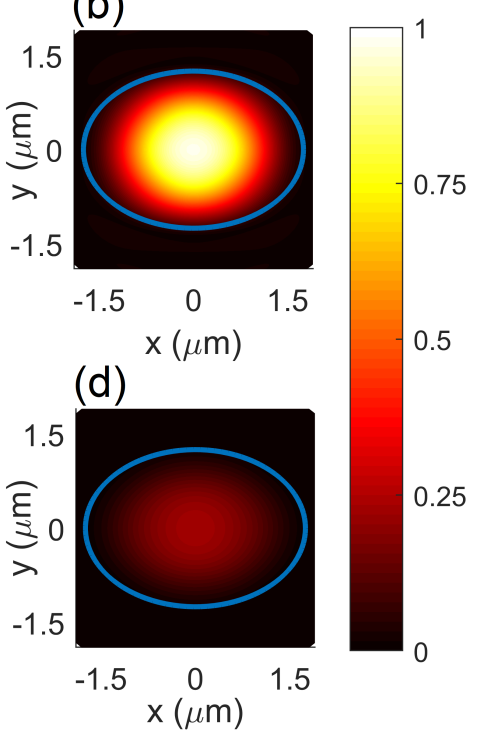

FIG. 3. Electric near-field distributions of the circular $(a, c)$ and the elliptical $\left(D_{x}=3.5 \mu \mathrm{m}\right)$ micropillar cavity structure $(\mathrm{b}, \mathrm{d})$. The near field is computed at the top of the structure for a point dipole emitting at the $\lambda_{0}=895 \mathrm{~nm}$ design wavelength. The first row (a,b) presents the co-polarized $\mathrm{X}$ cavity mode fields, while the second row (c,d) shows the cross-polarized Y mode fields.

preserved, we may expect a similarly high $\eta \sim 0.99$. To keep the mode volume (roughly) constant, we introduce ellipticity $\left(e=1-D_{y} / D_{x}\right)$ to the structure while keeping the sum of the major axis $\left(D_{x}\right)$ and the minor axis $\left(D_{y}\right)$ constant at $6 \mu \mathrm{m}$. For each set of considered semi-diameters, the layer thicknesses are chosen as discussed above such that the resonance of the X-polarized mode is maintained at the design wavelength $\lambda_{0}=895 \mathrm{~nm}$.

We now present the power $P_{\text {cav }}^{\mathrm{Y}}$ coupled to the crosspolarized $Y$ mode in Fig. 2 as function of $\lambda$. For a circular micropillar with $e=0\left(D_{x}=D_{y}\right.$, black curve), the peak $P_{\text {cav }}^{\mathrm{Y}}$ power is obtained at the design wavelength $\lambda_{0}$, since the power spectra for the two polarizations in this case are identical. However, increasing the ellipticity by increasing the length $D_{x}$ of the major axis shifts the cross-polarized Y mode towards shorter wavelengths leading to a decrease of $P_{\mathrm{cav}}^{\mathrm{Y}}$ at the design wavelength. A slight ellipticity of $e=0.13\left(D_{x}=3.2 \mu \mathrm{m}\right.$, red curve) results in a relative suppression of the cross-polarized $\mathrm{Y}$ mode of $\sim 2 \mathrm{~dB}$ at the design wavelength, whereas a larger ellipticity, such as $e=0.29\left(D_{x}=3.5 \mu \mathrm{m}\right.$, yellow curve), allows for a suppression $\sim 13 \mathrm{~dB}$. To visualize the suppression effect of the ellipticity, we show in Fig. 3(a-d) the near-field distributions computed in the horizontal plane just above the top DBR section. The X polarized near fields are presented for the circular and an elliptical pillar in Figs. 3(a,b) respectively, whereas the corresponding $\mathrm{Y}$ polarized near fields are shown in Figs. 3(c,d). The Y-polarized near field for the elliptical cross-section in Fig. 3(d) is suppressed at the design wavelength, and we observe that this leads to a relative reduction of its field intensity compared to the co-polarized field in Figs. 3(b) and the fields for the circular configuration.

The computed figures of merit for elliptical micropillar cavities with ellipticities from 0 to 0.8 (corresponding to major axes $D_{x}$ between $3 \mu \mathrm{m}$ and $5 \mu \mathrm{m}$ ) are shown in Fig. 4. Again, the layer thicknesses are chosen for each set of $D_{x}$ and $D_{y}$ values to always maintain the resonance peak for the co-polarized $X$ mode at the design wavelength of $\lambda_{0}=895 \mathrm{~nm}$. We find in Fig. 4(a) that the increasing ellipticity hardly affects the Q factor for the co-polarized $\mathrm{X}$ mode, whereas a slight reduction for the cross-polarization occurs due to the layer thicknesses not being optimized for the Y mode. The stability in the co-polarized Q factor ensures that the high degree of indistinguishability of the circular micropillar in the presence of phonon-induced decoherence is maintained for increasing ellipticity. The Purcell factor $F^{\mathrm{Y}}\left(\lambda_{0}\right)$ for the cross-polarized $Y$ mode evaluated at the design wavelength decreases quickly in Fig. 4(b) with increasing ellipticity as the resonance for the $\mathrm{Y}$ mode is blue-shifted away from the design wavelength as discussed earlier. On the other hand, the Purcell enhancement for the $\mathrm{X}$ mode is increased as its mode volume is not exactly conserved by the requirement $D_{x}+D_{y}=6 \mu \mathrm{m}$ but instead is slightly reduced with increasing ellipticity.

The spontaneous emission factor $\beta$ is identical for the two polarizations for the circular geometry as shown in Fig. 4(c). However, the drop in $F^{\mathrm{Y}}\left(\lambda_{0}\right)$ with increasing ellipticity leads to a reduction of $P_{\mathrm{T}}^{\mathrm{y}}$ in the denominator of Eq. 3, and in turn to an increase in $\beta^{\mathrm{X}}$ towards unity. Correspondingly, the drop in $F^{\mathrm{Y}}\left(\lambda_{0}\right)$ results in a decrease of $\beta^{\mathrm{Y}}$ towards 0 . This selective increase of $\beta^{\mathrm{X}}$ and suppression of $\beta^{\mathrm{Y}}$ with ellipticity is the central mechanism for establishing polarization control using an elliptical cross section. The transmission $\gamma$ from the cavity to the lens is shown in Fig. 4(d). For both polarizations, the maximum transmission is obtained for the circular geometry where the overlap with the Gaussian profile in the far field is maximum. As the ellipticity increases, the transmission de- 

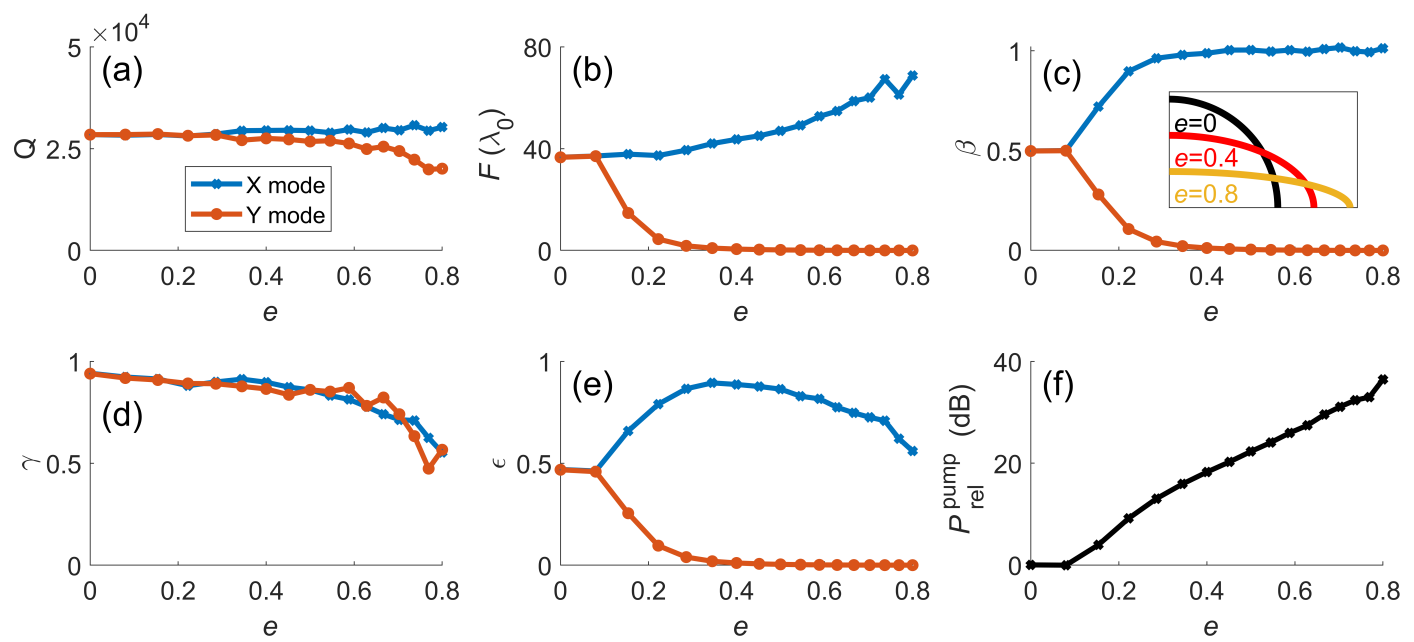

FIG. 4. Figures of merit for the co-polarized X (blue curves) and cross-polarized Y (red curves) modes: The Q factor (a), the Purcell factor $F$ (b) evaluated at the design wavelength $\lambda_{0}$, the spontaneous emission $\beta$ factor (c), the collection efficiency $\gamma$ (d) for a 0.82 NA lens and the overall $\varepsilon$ efficiency (e) as function of ellipticity $\left(e=1-D_{y} / D_{x}\right)$, where the sum of the semi-axes is kept constant such that $D_{x}+D_{y}=6 \mu \mathrm{m}$. The relative increase in required pump power (see main text) is presented in (f). The number of DBR layer pairs are $N_{\text {top }}=21$ and $N_{\text {bottom }}=40$. The inset shows quarter cross-sections for $e=0$ (black), $e=0.4$ (red) and $e=0.8$ (orange).

creases due to a deterioration of the overlap of the elliptical modes with the perfect circular Gaussian profile, and this reduction leads to a trade-off between the achievable $\beta^{\mathrm{X}}$ and $\gamma^{\mathrm{X}}$ for the elliptical micropillar design. The effect of this tradeoff is observed for the overall source efficiency $\varepsilon$ presented in Fig. 4(e), where we observe that a maximum efficiency of $\varepsilon^{\mathrm{X}} \sim 0.90$ is obtained for $e=0.35$ corresponding to $D_{x}=$ $3.64 \mu \mathrm{m}$. Moreover, the polarized efficiency remains above 0.85 for ellipticity between 0.27 and 0.52 corresponding to $D_{x} \in[3.47,4.1] \mu \mathrm{m}$.

An important practical consideration when implementing the elliptical cross-section is the increase in required pump power as the QD is pumped using $y$-polarized light. The relative increase $P_{\text {rel }}^{\text {pump }}$ between the pump power needed for achieving saturation of the QD at the design wavelength for the circular geometry and for the elliptical pillar is given by

$$
P_{\mathrm{rel}}^{\mathrm{pump}}(e)=\frac{P_{\mathrm{cav}}^{\mathrm{Y}}(e=0)}{P_{\mathrm{cav}}^{\mathrm{Y}}(e)}
$$

and is plotted in Fig. 4(f), where we observe that $P_{\text {rel }}^{\text {pump in- }}$ creases exponentially with the ellipticity. For $e=0.8$, a pump power increase of four orders of magnitude is required to saturate the QD, and this rapid exponential increase in pump power represents a significant drawback of elliptical crosssection strategy for implementing polarization control.

We now discuss the strengths and weaknesses of the elliptical micropillar design strategy used in the cross-polarized resonant excitation scheme. The elliptical cross-section is straight-forward to implement and allows for an increase of the spontaneous emission $\beta^{\mathrm{X}}$ of the desired co-polarized $\mathrm{X}$ mode towards unity. However, a trade-off occurs due to the reduced overlap of the elliptical mode profile with the Gaussian in the far field. Additionally, the same suppression mechanism which ensures high polarization control also increases the required pump power. For the elliptical micropillar design under consideration, a maximum polarized efficiency of $\varepsilon^{\mathrm{X}} \sim$ 0.90 is obtained for $e=0.35$, and here the wavelength splitting of $0.12 \mathrm{~nm}$ is $\sim 4$ times smaller and the relative increase in pump power of $16 \mathrm{~dB}$ is only $5 \mathrm{~dB}$ larger than in a recent experimental demonstration ${ }^{12}$. While a complete optimization of both $\varepsilon$ (without the $D_{x}+D_{y}=6 \mu \mathrm{m}$ constraint) and $\eta$ from scratch is of interest, it is beyond this Letter. Instead, our work exploits the findings of Ref. 17, where explicit calculations of achievable photon indistinguishability in the presence of phonon-induced decoherence were performed. Since the indistinguishability depends only on the $\mathrm{Q}$ factor and the mode volume, we may expect a similar high indistinguishability with $\eta>0.99$ as in Ref. 17 for elliptical micropillars with similar Q factors and mode volumes of the X mode. While the intrinsic $Q$ factor is largely unaffected by introduction of the elliptical cross-section, sidewall imperfection in realistic devices is known ${ }^{18}$ to cause scattering loss and a reduction in $\mathrm{Q}$. However, we note that the reduced $0.85 \mathrm{Al}$ fraction in the $\mathrm{Al}_{0.85} \mathrm{Ga}_{0.15}$ As layer considered here strongly suppresses oxidation as compared to pure AlAs thus reducing sidewall imperfection. Also, extrinsic loss in elliptical micropillars affects the $\mathrm{X}$ mode less ${ }^{12,14}$ than the $\mathrm{Y}$ mode, and with these observations we leave the more detailed investigation of the effect of extrinsic loss to a follow-up work. Now, while Fig. 4(b) indicates a decrease in mode volume up to a factor of $\sim 2$ for $e=0.8\left(D_{x}=5 \mu \mathrm{m}\right)$, Fig. 4(b) in Ref. 17 shows that the indistinguishability $\eta$ for $N_{\text {top }}=21$ is stable in the $D \in[2,3]$ $\mu \mathrm{m}$ diameter regime, and we may thus expect $\eta>0.99$ in the entire $D_{x} \in[3,5] \mu \mathrm{m}$ range considered in this work. While we have showed that a product $\varepsilon \eta \sim 0.89$ may be achieved using the elliptical micropillar design, the trade-off between achievable $\beta^{\mathrm{X}}$ and $\gamma^{\mathrm{X}}$ represents a bottleneck for the elliptical micropillar SPS and ways of increasing $\varepsilon \eta$ beyond 0.89 
are not obvious. One may envision that the trade-off could be overcome by implementing a new vertical geometry featuring a transition from an elliptical cross-section at the position of the QD to a circular (or only slightly elliptical) cross-section at the top of the DBR. Such a transition could naturally be implemented using the recently proposed "hourglass" geometry ${ }^{38}$ which could potentially allow for a performance $\varepsilon \eta \sim$ far beyond 0.89 .

In conclusion, we have performed a numerical optimization of the elliptical micropillar geometry, which allows for polarization control such that the $50 \%$ barrier of the crosspolarization scheme used in resonant excitation may be overcome. We have demonstrated that a polarized efficiency of 0.90 can be achieved combined with a modest increase in required pump power of $16 \mathrm{~dB}$. We have shown that the geometry is compatible with photon indistinguishabilities above 0.99 . The numerically predicted single-photon source performance in this work presents a significant step forward as compared to the state-of-the-art.

The authors acknowledge support from Villum Fonden (VKR Center of Excellence NATEC-II, grant 8692), from the European Research Council (ERC-CoG "UNITY", grant 865230) and from the Independent Research Fund Denmark (grant DFF-9041-00046B).

\section{DATA AVAILABILITY}

The data that support the findings of this study are available within the article.

${ }^{1}$ N. Gregersen, P. Kaer, and J. Mørk, "Modeling and design of highefficiency single-photon sources," IEEE J. Sel. Top. Quantum Electron. 19, 9000516 (2013).

${ }^{2}$ N. Gregersen, D. P. S. McCutcheon, and J. Mørk, "Single-photon sources," in Handbook of optoelectronic device modeling and simulation Vol. 2, edited by J. Piprek (CRC Press, Boca Raton, 2017) Chap. 46, pp. 585-607. ${ }^{3}$ P. G. Kwiat, K. Mattle, H. Weinfurter, A. Zeilinger, A. V. Sergienko, and Y. Shih, "New high-intensity source of polarization-entangled photon pairs," Phys. Rev. Lett. 75, 4337-4341 (1995).

${ }^{4}$ I. Aharonovich, D. Englund, and M. Toth, "Solid-state single-photon emitters," Nat. Photonics 10, 631-641 (2016).

${ }^{5}$ A. J. Shields, "Semiconductor quantum light sources," Nat. Photonics 1, 215-223 (2007), arXiv:0704.0403.

${ }^{6}$ W. L. Barnes, G. Björk, J. M. Gérard, P. Jonsson, J. A. E. Wasey, P. T. Worthing, and V. Zwiller, "Solid-state single photon sources: light collection strategies," Eur. Phys. J. D 18, 197-210 (2002).

${ }^{7}$ X. Ding, Y. He, Z.-C. Duan, N. Gregersen, M.-C. Chen, S. Unsleber, S. Maier, C. Schneider, M. Kamp, S. Höfling, et al., "On-demand single photons with high extraction efficiency and near-unity indistinguishability from a resonantly driven quantum dot in a micropillar," Phys. Rev. Lett. 116, 020401 (2016).

${ }^{8}$ N. Somaschi, V. Giesz, L. De Santis, J. C. Loredo, M. P. Almeida, G. Hornecker, S. L. Portalupi, T. Grange, C. Antón, J. Demory, C. Gómez, I. Sagnes, N. D. Lanzillotti-Kimura, A. Lemaítre, A. Auffeves, A. G. White, L. Lanco, and P. Senellart, "Near-optimal single-photon sources in the solid state," Nat. Photonics 10, 340-345 (2016).

${ }^{9}$ H. Ollivier, I. Maillette de Buy Wenniger, S. Thomas, S. C. Wein, A. Harouri, G. Coppola, P. Hilaire, C. Millet, A. Lemaître, I. Sagnes, O. Krebs, L. Lanco, J. C. Loredo, C. Antón, N. Somaschi, and P. Senellart, "Reproducibility of high-performance quantum dot single-photon sources," ACS Photonics 7, 1050-1059 (2020).

${ }^{10}$ Y.-M. He, H. Wang, C. Wang, M.-C. Chen, X. Ding, J. Qin, Z.-C. Duan, S. Chen, J.-P. Li, R.-Z. Liu, C. Schneider, M. Atatüre, S. Höfling, C.-Y. Lu, and J.-W. Pan, "Coherently driving a single quantum two-level system with dichromatic laser pulses,” Nat. Phys. 15, 941-946 (2019).
${ }^{11}$ S. E. Thomas, M. Billard, N. Coste, S. C. Wein, Priya, H. Ollivier, O. Krebs, L. Tazaïrt, A. Harouri, A. Lemaitre, I. Sagnes, C. Anton, L. Lanco, N. Somaschi, J. C. Loredo, and P. Senellart, "Bright polarized single-photon source based on a linear dipole," (2021), arXiv:2007.04330 [quant-ph].

${ }^{12}$ H. Wang, Y.-M. He, T.-H. Chung, H. Hu, Y. Yu, S. Chen, X. Ding, M.-C. Chen, J. Qin, X. Yang, et al., "Towards optimal single-photon sources from polarized microcavities," Nat. Photonics 13, 770-775 (2019).

${ }^{13}$ E. Moreau, I. Robert, L. Manin, V. Thierry-Mieg, J. Gérard, and I. Abram, "A single-mode solid-state source of single photons based on isolated quantum dots in a micropillar," Physica E Low Dimens. Syst. Nanostruct. 13, 418-422 (2002).

${ }^{14}$ B. Gayral, J. Gérard, B. Legrand, E. Costard, and V. Thierry-Mieg, "Optical study of GaAs/A1As pillar microcavities with elliptical cross section," Appl. Phys. Lett. 72, 1421-1423 (1998).

${ }^{15}$ S. Reitzenstein, C. Böckler, A. Löffler, S. Höfling, L. Worschech, A. Forchel, P. Yao, and S. Hughes, "Polarization-dependent strong coupling in elliptical high-Q micropillar cavities," Phys. Rev. B 82, 235313 (2010).

${ }^{16}$ A. Daraei, D. Sanvitto, J. Timpson, A. Fox, D. Whittaker, M. Skolnick, P. Guimarães, H. Vinck, A. Tahraoui, P. Fry, et al., "Control of polarization and mode mapping of small volume high Q micropillars," J. Appl. Phys. 102, 043105 (2007)

${ }^{17}$ B.-Y. Wang, E. V. Denning, U. M. Gür, C.-Y. Lu, and N. Gregersen, "Micropillar single-photon source design for simultaneous near-unity efficiency and indistinguishability," Phys. Rev. B 102, 125301 (2020).

${ }^{18}$ T. Rivera, J.-P. Debray, J. M. Gérard, B. Legrand, L. Manin-Ferlazzo, and J. L. Oudar, "Optical losses in plasma-etched AlGaAs microresonators using reflection spectroscopy," Appl. Phys. Lett. 74, 911-913 (1999).

${ }^{19}$ J. Claudon, J. Bleuse, N. S. Malik, M. Bazin, P. Jaffrennou, N. Gregersen, C. Sauvan, P. Lalanne, and J.-M. Gérard, "A highly efficient single-photon source based on a quantum dot in a photonic nanowire," Nat. Photonics 4, 174-177 (2010).

${ }^{20}$ M. E. Reimer, G. Bulgarini, N. Akopian, M. Hocevar, M. B. Bavinck, M. A. Verheijen, E. P. A. M. Bakkers, L. P. Kouwenhoven, and V. Zwiller, "Bright single-photon sources in bottom-up tailored nanowires," Nat. Commun. 3, 737 (2012), arXiv:1203.5676v1.

${ }^{21}$ M. Arcari, I. Söllner, A. Javadi, S. Lindskov Hansen, S. Mahmoodian, J. Liu, H. Thyrrestrup, E. H. Lee, J. D. Song, S. Stobbe, and P. Lodahl, "Near-unity coupling efficiency of a quantum emitter to a photonic crystal waveguide," Phys. Rev. Lett. 113, 093603 (2014), arXiv:1402.2081.

${ }^{22}$ M. Gschrey, R. Schmidt, J.-H. Schulze, A. Strittmatter, S. Rodt, and S. Reitzenstein, "Resolution and alignment accuracy of low-temperature in situ electron beam lithography for nanophotonic device fabrication," J. Vac. Sci. Technol. B 33, 021603 (2015).

${ }^{23}$ Y.-M. He, Y. He, Y.-J. Wei, D. Wu, M. Atatüre, C. Schneider, S. Höfling, M. Kamp, C.-Y. Lu, and J.-W. Pan, "On-demand semiconductor singlephoton source with near-unity indistinguishability," Nat. Nanotechnol. 8, 213-217 (2013).

${ }^{24} \mathrm{P}$. Kaer, N. Gregersen, and J. Mørk, "The role of phonon scattering in the indistinguishability of photons emitted from semiconductor cavity QED systems," New J. Phys. 15, 035027 (2013).

${ }^{25}$ S. Gehrsitz, F. Reinhart, C. Gourgon, N. Herres, A. Vonlanthen, and H. Sigg, "The refractive index of Al x Ga 1- x As below the band gap: accurate determination and empirical modeling," J. Appl. Phys. 87, 78257837 (2000).

${ }^{26}$ N. Bonod, E. Popov, and M. Nevière, "Differential theory of diffraction by finite cylindrical objects," J. Opt. Soc. Am. A 22, 481-490 (2005).

${ }^{27}$ T. Häyrynen, J. R. de Lasson, and N. Gregersen, "Open-geometry Fourier modal method: Modeling nanophotonic structures in infinite domains," J. Opt. Soc. Am. A 33, 1298-1306 (2016).

${ }^{28}$ U. M. Gür, N. Gregersen, S. Arslanagić, and M. Mattes, "Modeling of quantum-dot elliptical nanowire single-photon sources," in 14th European Conference on Antennas and Propagation (EuCAP) (2020) pp. 1-3.

${ }^{29}$ L. Novotny and B. Hecht, Principles of nano-optics (Cambridge University Press, 2012).

${ }^{30}$ A. V. Lavrinenko, J. Lægsgaard, N. Gregersen, F. Schmidt, and T. Søndergaard, Numerical methods in photonics (CRC Press, 2014).

${ }^{31}$ M. Munsch, J. Claudon, J. Bleuse, N. S. Malik, E. Dupuy, J.-M. Gérard, Y. Chen, N. Gregersen, and J. Mørk, "Linearly polarized, single-mode spontaneous emission in a photonic nanowire," Phys. Rev. Lett. 108, 
077405 (2012).

${ }^{32}$ E. M. Purcell, "Spontaneous emission probabilities at radio frequencies," in Confined Electrons and Photons (Springer, 1995) pp. 839-839.

${ }^{33}$ A. E. Krasnok, A. P. Slobozhanyuk, C. R. Simovski, S. A. Tretyakov, A. N. Poddubny, A. E. Miroshnichenko, Y. S. Kivshar, and P. A. Belov, "An antenna model for the Purcell effect," Sci. Rep. 5, 12956 (2015).

${ }^{34} \mathrm{C}$. A. Balanis, Antenna theory: analysis and design (John Wiley \& Sons, 2016).

${ }^{35}$ M. Munsch, N. S. Malik, E. Dupuy, A. Delga, J. Bleuse, J.-M. Gérard, J. Claudon, N. Gregersen, and J. Mørk, "Dielectric GaAs antenna ensuring an efficient broadband coupling between an InAs quantum dot and a Gaussian optical beam,” Phys. Rev. Lett. 110, 177402 (2013).

${ }^{36}$ M. Munsch, N. S. Malik, E. Dupuy, A. Delga, J. Bleuse, J.-M. Gérard, J. Claudon, N. Gregersen, and J. Mørk, "Erratum: Dielectric GaAs An- tenna Ensuring an Efficient Broadband Coupling between an InAs Quantum Dot and a Gaussian Optical Beam [Phys. Rev. Lett. 110, 177402 (2013)]," Phys. Rev. Lett. 111, 239902 (2013).

${ }^{37}$ N. Gregersen, S. Reitzenstein, C. Kistner, M. Strauss, C. Schneider, S. Höfling, L. Worschech, A. Forchel, T. R. Nielsen, J. Mørk, et al., "Numerical and experimental study of the $\mathrm{Q}$ factor of high-Q micropillar cavities," IEEE J. Quantum Electron. 46, 1470-1483 (2010).

${ }^{38}$ A. D. Osterkryger, J.-M. Gérard, J. Claudon, and N. Gregersen, "Photonic "hourglass" design for efficient quantum light emission," Opt. Lett. 44, 2617-2620 (2019). 